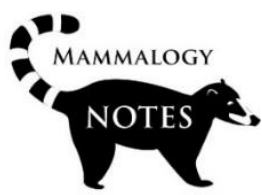

\title{
Depredación de Ctenosaura pectinata (Squamata: Iguanidae) por Lynx rufus (Carnivora: Felidae) en el Estado de Michoacán de Ocampo, México
}

\author{
Juan F. Charre-Medellín ${ }^{1,2 *}$ (iD), Tiberio C. Monterrubio-Rico2 (iD, \\ Eliazin Gómez-Cárdenas² (iD, María del Socorro Alvarado3 ${ }^{3}$, \\ Esteban Barragán-López ${ }^{4}$ (i) Rogelia Torres-Villa ${ }^{5}$ (D)
}

\begin{abstract}
1 Centro de Investigaciones en Geografía Ambiental, Universidad Nacional Autónoma de México, Antigua Carretera A Pátzcuaro 8701, Ex Hacienda San José de la Huerta, 58190, Morelia, Michoacán, México.

2 Laboratorio de Vertebrados Terrestres Prioritarios, Facultad de Biología, Universidad Michoacana de San Nicolás de Hidalgo, Gral. Francisco J. Múgica s/n A-1, Felicitas de Río, 58030, Morelia, Michoacán, México.

3 Centro de Investigación en Alimentación y Desarrollo A.C., Coordinación de Desarrollo Regional, Carretera Gustavo Enrique Astiazarán Rosas, N0. 46, 83304, Hermosillo, México.

4 Colegio de Michoacán A.C., Martínez de Navarrete 505, Las Fuentes, 59699, Zamora, Michoacán, México.

5 Unidad Académica de Estudios Regionales, Coordinación de Humanidades, Universidad Nacional Autónoma de México, Avenida Lázaro Cárdenas, Felicitas del Río s/n, Centro, 59510, Jiquilpan, Michoacán, México.
\end{abstract}

*Correspondencia: jcharre@ciga.unam.mx

\section{Resumen}

El gato montés (Lynx rufus Schreber 1777), es uno de los seis felinos que habitan en México, cuya distribución se asocia principalmente a zonas templadas del norte y centro del país. La dieta del gato montés se compone principalmente de pequeños mamíferos, aves y ocasionalmente reptiles. En este trabajo documentamos por primera vez la depredación de iguana negra (Ctenosaura pectinata) por gato montés y la inclusión en su dieta. De abril a junio de 2017 realizamos un muestreo sistemático con 20 cámaras trampa en el municipio de Tocumbo (Michoacán de Ocampo), en la región conocida como "Sierra de Jal-Mich". Con un esfuerzo de muestreo total de 1200 días/trampa, se obtuvo la primera evidencia de la presencia de la iguana negra en la dieta del gato montés. El registro se obtuvo en un sitio ubicado a $671 \mathrm{msnm}$, asociado a selva baja. Además, se registró la presencia de otras cinco especies de felinos. El consumo de reptiles por parte del gato montés parece aumentar en regiones donde la diversidad y tamaño corporal de los reptiles es mayor. Este reporte contribuye al conocimiento de la historia de vida del gato montés y posible coexistencia con otros felinos.

Palabras clave: Cámaras trampa, Dieta, Felidae, Ocelote.

Abstract

The bobcat (Lynx rufus Schreber 1777), is one of the six felines that inhabit Mexico, whose distribution is mainly associated with temperate zones in the north and center of the country. The bobcat diet is primarily made up of small mammals, birds, and occasionally reptiles. In this work, the 
predation of the black iguana (Ctenosaura pectinata) by bobcat and the inclusion in their diet are documented for the first time. From April to June 2017, we carried out a systematic sampling with 20 camera traps in Tocumbo municipality (Michoacán de Ocampo), in the region known as "Sierra de Jal-Mich". With a total sampling effort of 1200 days/trap, the first evidence of the presence of the black iguana in the diet of the bobcat was obtained. The record was obtained at a site located 671 masl, associated with the dry forest. In addition, we record five other wild cat species. The consumption of reptiles by the bobcat seems to increase in regions where the diversity and body size of reptiles is greater. This report contributes to the knowledge of the life history of the wild cat and the possible coexistence with other wild cats.

Key words: Camera traps, Diet, Felidae, Ocelot.

El gato montés o lince rojo (Lynx rufus Schreber 1777), es un felino de talla mediana con una amplia distribución en México y Norteamérica. Es el tercer felino más grande entre las seis especies que habitan en México (Lariviere \& Walton 1997; Brockmeyer \& Clark 2007; Ceballos et al. 2010). En México, la presencia de este felino se asocia principalmente a zonas templadas y desérticas de norte y centro de México, hasta el Istmo de Tehuantepec en Oaxaca (Lariviere \& Walton 1997; Medellín \& Bárcenas 2010). Además, el gato montés es el felino más estudiado en Norteamérica, donde su distribución, ecología y dieta ha sido documentada ampliamente, principalmente en los Estados Unidos (McCord \& Cardoza 1982; Anderson \& Lovallo 2003; Brodie 2009).

De acuerdo con la mayoría de los estudios sobre la dieta del gato montés, se ha observado que esta se basa principalmente en mamíferos, siendo los lagomorfos y los roedores sus principales presas (Nussbaum \& Maser 1975; Salas 1987; Delibes et al. 1997; Aranda et al. 2002; Brockmeyer \& Clark 2007; Medellín \& Bárcenas 2010; Larson et al. 2015). Sin embargo, existen estudios que reportan en la dieta de los gatos montés, aves (Litvaitis 1981; Delibes et al. 1997; Tewes et al. 2002; Booth-Binczik et al. 2013; Larson et al. 2015) y en menor medida reptiles (Litvaitis 1981; Delibes \& Hilaldo 1987; Delibes et al. 1997). Por tal motivo, en este trabajo reportamos por primera vez la inclusión de la iguana negra (Ctenosaura pectinata) en la dieta del gato montés, a partir de una fotografía que muestra la depredación de la iguana negra por parte de un gato montés.

De abril a junio de 2017 realizamos un muestreo sistemático con cámaras trampa, en el que se colocaron 20 cámaras marca Cuddeback Digital ${ }^{\circledR}$ de 20 megapixeles, en varias localidades del suroeste del municipio de Tocumbo (Michoacán de Ocampo), en la región conocida como "Sierra de JalMich", limítrofe entre Jalisco y Michoacán en el occidente de México (Figura 1). Las cámaras se instalaron asociadas a manantiales, cañadas y senderos con evidencia de presencia de mamíferos. Se programaron para que se activaran cada 3 minutos y tomaran 3 fotos por evento. La vegetación y uso de suelo presente en el área de estudio incluye áreas agropecuarias, selva baja caducifolia, bosque de encino y en menor cantidad remanentes de bosque de pino en las zonas más altas (Mas et al. 2017).

Con un esfuerzo de muestreo total de 1200 días/trampa, se obtuvo la primera evidencia de la presencia de la iguana negra en la dieta del gato montés (Figura 2). El registro se obtuvo el 23 de abril a las 13h26, en un sitio ubicado a $671 \mathrm{msnm}$, asociado a selva baja caducifolia (19,5413, -102,7116). Además de la presencia de gato montés, se registraron cinco especies de felinos: puma (Puma concolor), jaguarundi (Herpailurus yagouaroundi), tigrillo (Lepoardus wiedii), ocelote (Leopardus pardalis) y jaguar (Panthera onca) (Charre-Medellín et al. 2018). Durante el monitoreo no se registró ninguna especie de lagomorfo. 


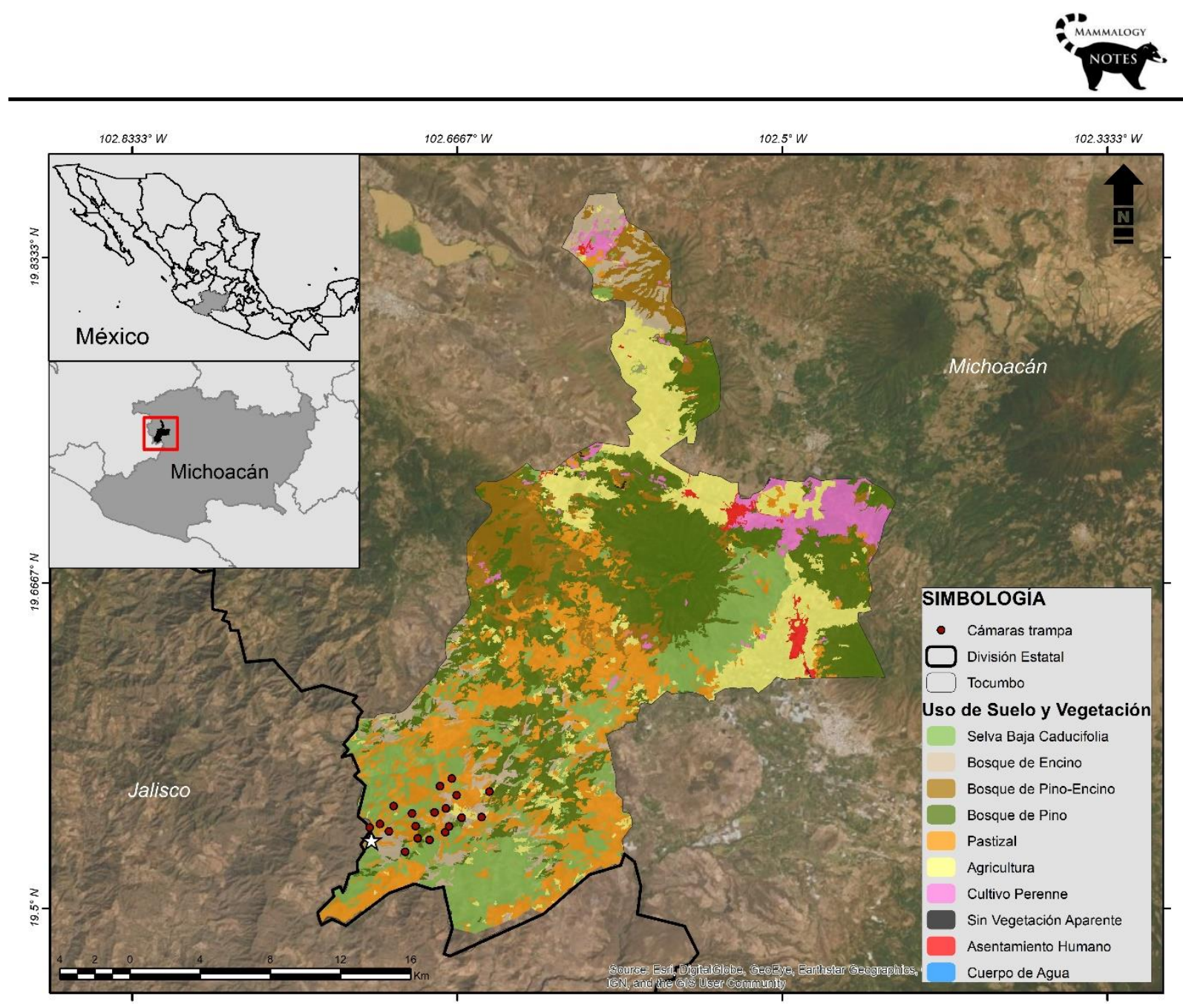

FIGURA 1. Ubicación del primer registro de la depredación (estrella blanca) de iguana negra (Ctenosaura pectinata) por un gato montés (Lynx rufus) en México.

La dieta del gato montés presenta una variación estacional y regional a lo largo de su extenso rango de distribución, de manera general, se ha observado que se comporta como un depredador que captura a sus presas dependiendo de la abundancia (Delibes \& Hilaldo 1987; Lariviere \& Walton 1997; Anderson \& Lovallo 2003; Hansen 2007). En este sentido, la inclusión de la iguana negra en la dieta de gato montés, se suma a la iguana del desierto (Dipsosaurus dorsalis) y a la iguana de cola espinosa (Ctenosaura hemilopha), reportadas por Delibes et al. (1997) en Baja California Sur, donde estas especies son los reptiles más abundantes en esa región.

Si bien, la depredación de reptiles por los gatos montés se ha considerado inusual dentro de su rango de distribución, el consumo de reptiles por estos felinos parece aumentar a medida que disminuye la latitud, debido a que la diversidad y tamaño corporal de los reptiles aumenta a menor latitud, lo que parece ser más benéfico en términos energéticos para estos felinos (Delibes et al. 1997).

Hasta el día de hoy, la presencia del gato montés en el estado de Michoacán, se documentaba en zonas templadas de las ecorregiones del Bajío y Eje Neovolcánico Transversal, por arriba de los 1500 msnm, donde la riqueza y abundancia de competidores potenciales 
(felinos) es baja y la abundancia de presas potenciales (lagomorfos) es alta (Charre-Medellín et al. 2015). Por lo que el registro de gato montés reportado en este trabajo, además de estar asociado con la presencia de otras cinco especies de felinos, también representa el primero en selva baja caducifolia y el de menor elevación (671 msnm) para el estado de Michoacán, lo cual permite que exista una mayor competencia por recursos alimenticios, principalmente con el ocelote, al ser una especie que compite con el gato montés en las áreas donde coexisten (Booth-Binczik et al. 2013).

A diferencia del gato montés, la iguana negra es una de las principales presas del ocelote en regiones tropicales de México (Villa-Meza et al. 2002), por lo que la depredación de reptiles por el gato montés puede deberse a la baja abundancia local de sus principales presas potenciales como lagomorfos o roedores, optimizando su dieta al incrementar la depredación de presas alternativas como la iguana negra (Baker et al. 2001). La hora del día en la que ocurrió la depredación de la iguana negra, sugiere que el gato montés y los ocelotes cohabitan difiriendo en los horarios de actividad, ya que el gato montés de manera general se encuentra más activo durante la noche (Lariviere \& Walton 1997). El traslape de nicho y preferencia de hábitos alimenticios de estas dos especies de felinos, es un tema que debe ser estudiado en las zonas tropicales de México donde coexisten.

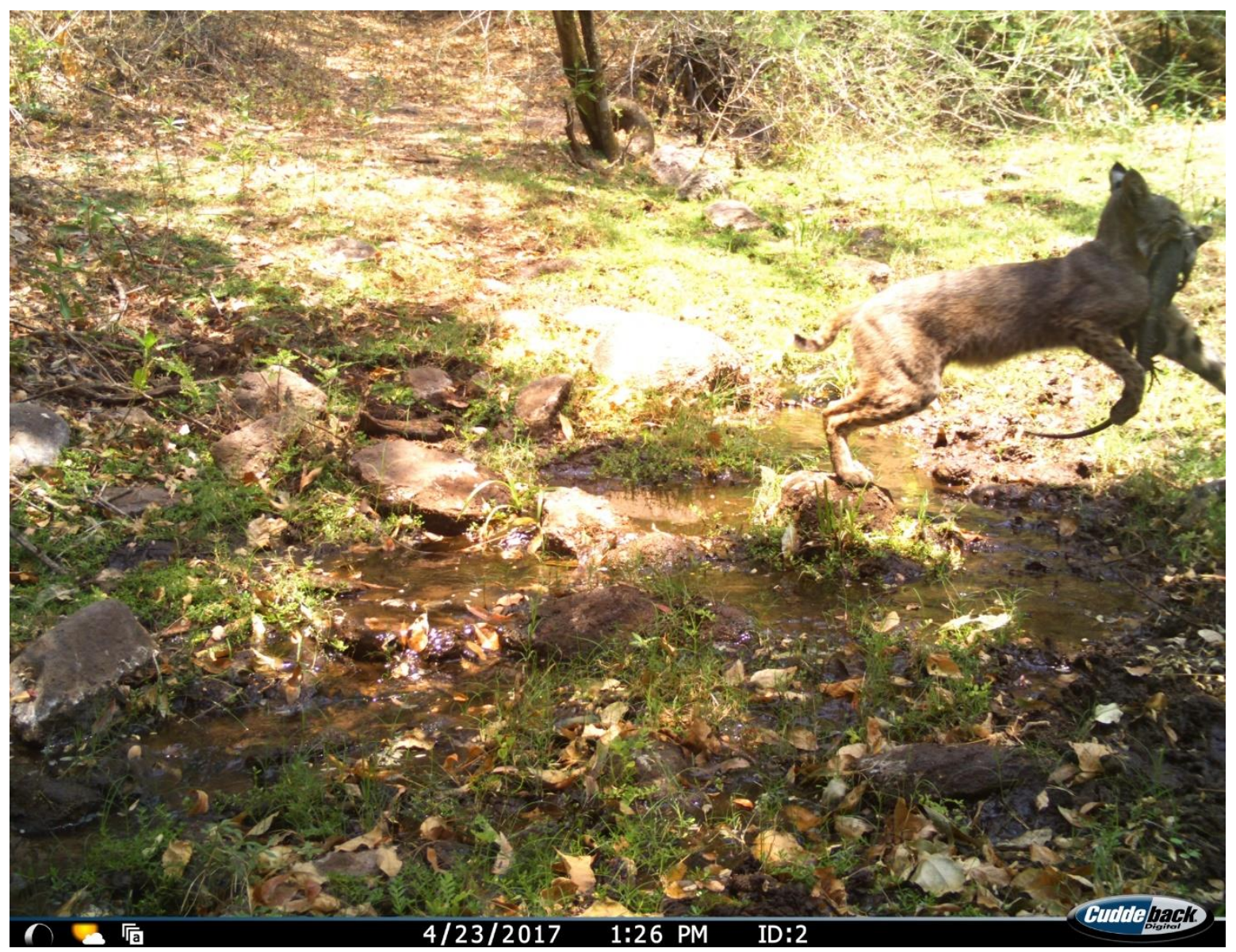

FIGURA 2. Evento de depredación de iguana negra (Ctenosaura pectinata) por un gato montés (Lynx rufus) en México. 


\section{AGRADECIMIENTOS}

Al Colegio de Michoacán, donde se iniciaron los esfuerzos de conservación de la vida silvestre en la región. Agradecemos el interés, la participación y la asistencia en campo por parte de los habitantes de las comunidades de Palo Bobo, Los Desmontes, El Rodeo y El Santuario. A la Coordinación de Investigación Científica de UMSNH por financiar parcialmente esta investigación. Charre-Medellín agradece al CONACYT por la beca otorgada (239248) y a la Dirección General de Asuntos del Personal Académico (DGAPA), UNAM por la beca posdoctoral otorgada.

\section{REFERENCIAS}

Anderson E, Lovallo M. 2003. Bobcat and lynx. Lynx rufus and L. canadensis. In: Feldhamer G, Thompson B, Chapman J, editors. Wild Mammals of North America: Biology, Management, and Conservation. Johns Hopkins University Press, Baltimore, Maryland. p. 758-786.

Aranda M, Rosas O, Ríos J, García N. 2002. Análisis comparativo de la alimentación del gato montés (Lynx rufus) en dos diferentes ambientes de México. Acta Zoológica Mexicana 87:99-109.

Baker L, Warren R, Diefenbach D, James W, Conroy M. 2001. Prey selection by reintroduced Bobcats (Lynx rufus) on Cumberland Island, Georgia. The American Midland Naturalist 145:80-93. https://doi.org/https://doi.org/10.1674/0003-0031(2001)145[0080:PSBRBL]2.0.C0;2

Booth-Binczik S, Bradley R, Thompson C, Bender L, Huntley J, Harvey J, Laack L, Mays J. 2013. Food Habits of Ocelots and potential for competition with Bobcats in Southern Texas. The Southwestern Naturalist 58:403-410. https:// doi.org/https:/ / doi.org/10.1894/0038-4909-58.4.403

Brockmeyer KJ, Clark WR. 2007. Fall and Winter Food Habits of Bobcats (Lynx rufus) in Iowa. J. The Journal of the lowa Academy of Science 114:40-43. https://doi.org/https://scholarworks.uni.edu/jias/vol114/iss1/6

Brodie J. 2009. Is research effort allocated efficiently for conservation? Felidae as a global case study. Biodiversity and Conservation 18:2927-2939.

Ceballos G, List R, Medellín R, Bonacic C, Pacheco J. 2010. Los felinos de américa. Cazadores sorprendentes. TELMEX, México.

Charre-Medellín J, Monterrubio-Rico T, Guido-Lemus D, Mendoza E. 2015. Patrones de distribución de felinos silvestres (Carnivora: Felidae) en el trópico seco del Centro-Occidente de México. Revista de Biología Tropical 63:783-797.

Charre-Medellín JF, Barragán-López E, Torres-Villa R, Alvarado S, Monterrubio-Rico TC, GutiérrezBarragán A. 2018. Jaguar in the Tepalcatepec basin in central-western Michoacán, México. Therya 9:191-194. https:// doi.org/10.12933/therya-18-573

Delibes M, Hilaldo F. 1987. Food Habits of the Bobcat in Two Habitats of the Southern Chihuahuan Desert. The Southwestern Naturalist 32:457-461. https:// doi.org/10.2307/3671478

Delibes M, Zapata S, Blázquez M, Rodríguez-Estrella R. 1997. Seasonal food habits of bobcats (Lynx rufus) in subtropical Baja California. Canadian Journal of Zoology 75:478-483. https://doi.org/10.1139/297-058

Hansen K. 2007. Bobcat, master of survival. Oxford University Press, New York, E.U.A.

Lariviere S, Walton L. 1997. Lynx rufus. Mammalian Species 563:1-8. 
Larson R, Morin D, Wierzbowska I, Crooks K. 2015. Food habits of coyotes, gray foxes, and bobcats in a coastal southern California urban landscape. Western North American Naturalist 75:339347. https://doi.org/https://doi.org/10.3398/064.075.0311

Litvaitis J. 1981. A comparison of coyote and bobcat food habits in the Wichita Mountains, Oklahoma. Proc. Oklahoma Acad. Sci. 61:81-82.

Mas J, Lemoine-Rodríguez R, González R, López-Sánchez J, Piña-Garduño A, Herrera-Flores E. 2017. Evaluación de las tasas de deforestación en Michoacán a escala detallada mediante un método híbrido de clasificación de imágenes SPOT. Madera y Bosques 23:119-131.

McCord C, Cardoza J. 1982. Bobcat and Lynx. In: Chapman J, Feldhamer G, editors. Wild Mammals of North America. The John Hopkins University Press, Baltimore, Maryland. p. 728-766.

Medellín R, Bárcenas H. 2010. Estimación de la densidad y dieta del lince (Lynx rufus) en seis localidades de México. Informe final SNIB-CONABIO proyectos No. ES003 y ES009. Ciudad de México, México.

Nussbaum R, Maser C. 1975. Food Habits of the Bobcat, Lynx rufus, in the Coast and Cascade Ranges of Western Oregon in Relation to Present Management Policies. Northwest Science 49:261266.

Salas M. 1987. Hábitos alimenticios de la zorra, coyote y gato montés en la Sierra Tarasca. Ciencia Forestal 12:117-132.

Tewes ME, Mock JM, Young JH. 2002. Bobcat predation on quail, birds, and mesomammals. Natl. Quail Symp. Proc. 5:65-69.

Villa-Meza A, Martínez-Meyer E, López-González C. 2002. Ocelot (Leopardus pardalis) Food Habits in a Tropical Deciduous Forest of Jalisco, Mexico. American Midland Naturalist 148:146-154.

Editor: Angela V. Rojas-Rojas

Recibido 2020-05-01

Revisado 2020-05-10

Aceptado 2020-05-15

Publicado 2020-06-26 